\title{
Escherichia coli Endocarditis Presenting With Septic Shock in an Immunocompetent Female Patient
}

\author{
Christos Davoulos ${ }^{\mathrm{a}}$, Maria Lagadinou ${ }^{\mathrm{a}}$, Athanasios Moulias ${ }^{\mathrm{b}}$, Christos Triantos $^{\mathrm{a}}$, \\ Nikolaos Koutsogiannis $^{\mathrm{b}}$, Markos Marangos ${ }^{\mathrm{c}}$, Stelios F. Assimakopoulos ${ }^{\mathrm{c}, \mathrm{d}}$
}

\begin{abstract}
Escherichia coli (E. coli) is a rare cause of infective endocarditis, despite being a common cause of bacteremia. E. coli endocarditis affects most frequently immunocompromised elderly women, especially those with diabetes mellitus. We present a case of a 78-year-old female immunocompetent patient, presenting with septic shock and multiple organ dysfunction syndrome. E. coli was isolated in all sets of blood cultures and in urine culture and a contrast-enhanced abdominal computed tomography (CT) scan revealed spleen and left kidney infracts. Transthoracic echocardiography revealed a large $(>15 \mathrm{~mm})$ mobile mass on the atrial side of the posterior mitral valve leaflet. The patient was initially treated with intravenous ceftriaxone and ciprofloxacin for 2 weeks with successful clinical response and clearance of bacteremia, was then subjected to valve replacement (with isolation of $E$. coli from replaced valve cultures) and continued antibiotic therapy for additional 4 weeks postoperatively. E. coli has emerged in recent years as an important cause of bacteremia, especially in the elderly. In selected patients, as those with persistent Gram-negative bacteremia or severe sepsis/septic shock, echocardiography is of paramount importance for the diagnosis of Gram-negative endocarditis and should be included in our diagnostic algorithm of patient's evaluation.
\end{abstract}

Keywords: Infective endocarditis; E. coli; Bacteremia; Gram-negative endocarditis; Septic shock

\section{Introduction}

Infective endocarditis (IE) is a life-threatening disease that

Manuscript submitted August 27, 2019, accepted September 6, 2019

aDepartment of Internal Medicine, School of Medicine, University of Patras, 26504, Rion-Patras, Greece

bDepartment of Cardiology, School of Medicine, University of Patras, 26504, Rion-Patras, Greece

${ }^{c}$ Division of Infectious Diseases, Department of Internal Medicine, School of Medicine, University of Patras, 26504, Rion-Patras, Greece

${ }^{\mathrm{d}}$ Corresponding Author: Stelios F. Assimakopoulos, Division of Infectious Diseases, Department of Internal Medicine, School of Medicine, University of Patras, Patras 26504, Greece. Email: sassim@upatras.gr

doi: https://doi.org/10.14740/cr940 results in cardiac dysfunction and development of metastatic infections. It remains a severe clinical condition characterized by high rates of morbidity and mortality [1].

Gram-positive bacteria cause the vast majority of IE cases; however, nowadays Gram-negative bacilli (GNB) have gained attention owing to their increasing spread and its associated high morbidity and mortality rates and health care costs $[1,2]$. Contemporary published experience in Gram-negative IE is limited and the larger body of data derives from studies on IE due to the so-called "HACEK" group, that includes the genuses Haemophilus, Aggregatibacter, Cardiobacterium, Eikenella and Kingella. Escherichia coli (E. coli) is a rare cause of IE occurring most frequently in immunocompromised elderly women, especially those with diabetes mellitus [3].

Among 2,761 patients with definite IE enrolled in the International Collaboration on Infective Endocarditis Prospective Cohort Study (ICE-PCS) database, non-HACEK Gramnegative organisms were the cause in 49 (1.8\%): E. coli was the cause in $29 \%$ of them $(0.51 \%)$ [4]. In another multicenter prospective study conducted from 2004 to 2011, from the Italian Endocarditis Study Group, 58 cases of non-HACEK Gram-negative endocarditis amongst 1,722 patients were reported $(3.3 \%)$ and E. coli was the etiologic factor in 18 patients (1\%) [2]. Urinary tract infection was the most common source of infection, whilst other risk factors for acquisition of non-HACEK GNB endocarditis included the presence of immunosuppression or cardiac implantable electronic device [2]. In the aforementioned study, the overall in-hospital mortality was $13.8 \%$ (all deaths were attributable to IE), while 1-year cumulative mortality was $30.6 \%$, with a cumulative IE-related 1 -year mortality of $22.4 \%$.

We present herein a rare case of E. coli endocarditis, developed in an immunocompetent female patient, presenting with septic shock and multiple septic emboli in kidneys and spleen.

\section{Case Report}

A 78-year-old female patient was admitted to the University Hospital of Patras, Western Greece, with septic shock and multiple organ dysfunction syndrome with depressed mental function, cardiac failure, respiratory alkalosis and disseminated intravascular coagulation (DIC). Patient's relatives reported that the patient presented fever up to $40{ }^{\circ} \mathrm{C}$ with rigor of 3 days' 
Table 1. Laboratory Tests on Admission and After 2 Weeks of Antimicrobial Therapy

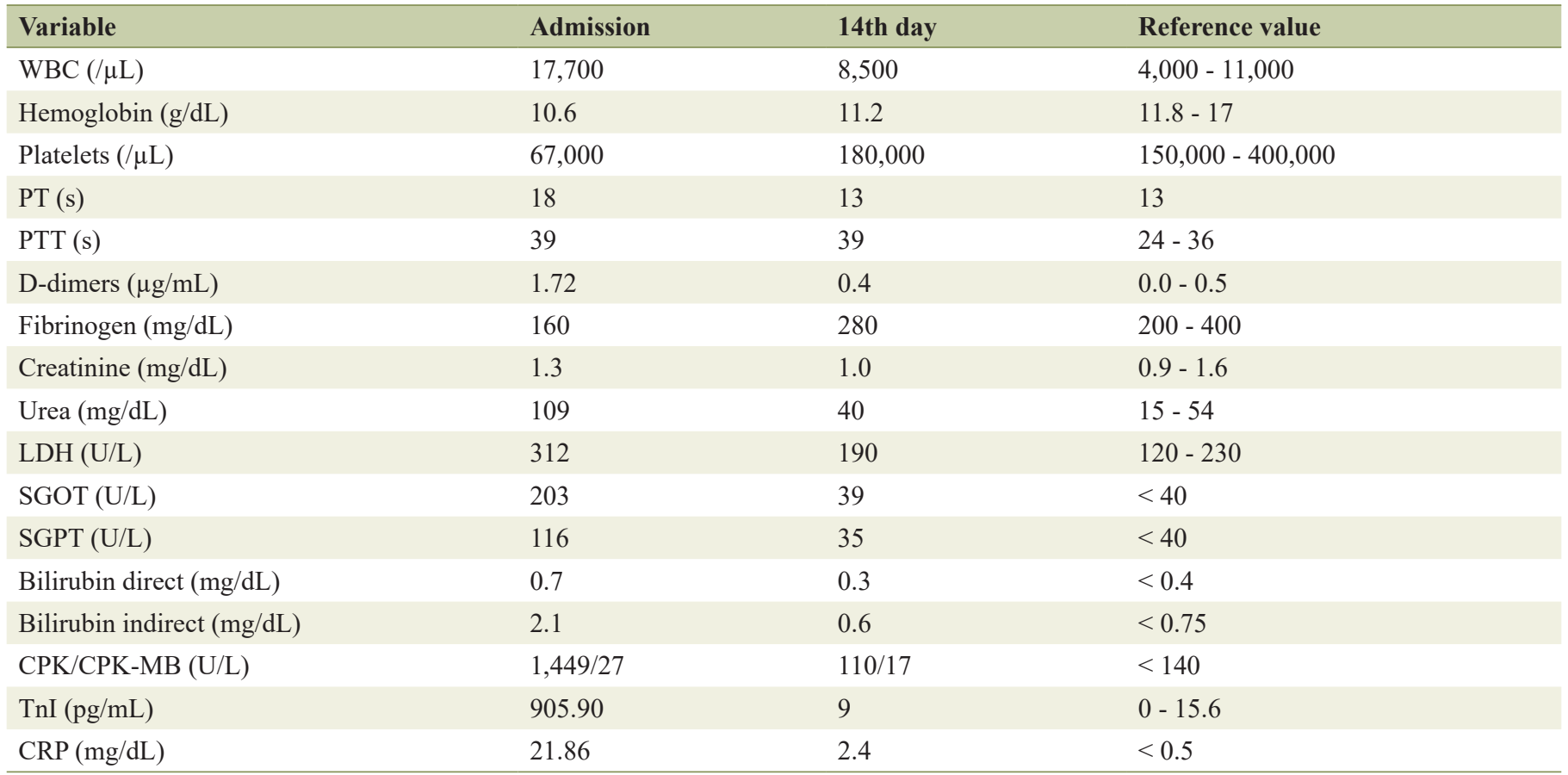

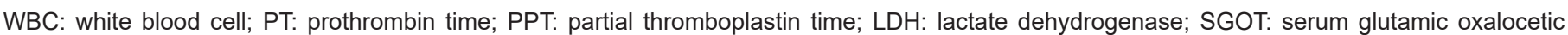
transaminase; SGPT: serum glutamic pyruvic transaminase; CPK: creatinine phosphokinase; Tnl: troponin; CRP: C-reactive protein.

duration associated with dysuria, while had been prescribed second-generation cephalosporin for 2 days without any improvement. No other complaints were reported. Her past medical history was unrevealing.

On physical examination, body temperature was $39.4^{\circ} \mathrm{C}$, heart rate was 108 beats per minute, blood pressure was 80/40 $\mathrm{mm} \mathrm{Hg}$ and respiratory rate was 25 breaths per minute. There was mild peripheral cyanosis and oxygen saturation was $92 \%$ on room air. The patient presented with a Glasgow coma scale score of 11. Cardiac auscultation revealed the presence of an $\mathrm{S} 3$ gallop, while rales were heard over the lung bases. The abdomen was distended with diminished bowel sounds and there was a positive Giordano sign on percussion of the left flank area.

An arterial blood gas revealed $\mathrm{pH}$ of $7.48, \mathrm{PaCO}_{2}$ of 29 $\mathrm{mm} \mathrm{Hg}, \mathrm{PaO}_{2}$ of $62 \mathrm{~mm} \mathrm{Hg}$ and $\mathrm{HCO}_{3}{ }^{-}$of $21 \mathrm{mmol} / \mathrm{L}$. Electrocardiography (ECG) revealed sinus tachycardia without any ischemic changes. Furthermore, laboratory tests showed raised white blood cells count with polymorphonuclear predominance, anemia, thrombocytopenia, raised aspartate aminotransferase and alanine aminotransferase, indirect hyperbilirubinemia, elevated lactate dehydrogenase (LDH) and creatinine phosphokinase $(\mathrm{CPK})$ levels, elevated troponin (TnI) and C-reactive protein (CRP) (Table 1). Prothrombin time (PT) and partial thromboplastin time (PTT) were prolonged and d-dimers were increased, while fibrinogen was reduced. Microscopic examination of peripheral blood smear revealed several schistocytes and neutrophils' left shift. Urinalysis showed pyuria and positive nitrates. Blood and urine cultures were taken on admission. Chest X-ray showed redis- tribution of pulmonary vessels. A contrast-enhanced abdominal computed tomography (CT) scan revealed spleen and left kidney infracts (Fig. 1). The patient underwent transthoracic echocardiography, which revealed global left ventricular hypokinesia with left ventricular ejection fraction of $40 \%$ and a large $(>15 \mathrm{~mm})$ mobile mass on the atrial side of the posterior mitral valve leaflet (Fig. 2).

In parallel the patient was supported by administration of intravenous crystalloids $30 \mathrm{~mL} / \mathrm{kg}$. Loading doses of broadspectrum antibiotic therapy consisted of intravenous meropenem (2 g), intravenous vancomycin $(15 \mathrm{mg} / \mathrm{kg} \mathrm{BW})$ and gentamicin $(5 \mathrm{mg} / \mathrm{kg} \mathrm{BW})$, which were thereafter adjusted according to patient's estimated glomerular filtration rate (GFR). Due to persistent hypotension despite the initial crystalloid fluid challenge, norepinephrine was administered to raise mean arterial pressure at $65 \mathrm{~mm} \mathrm{Hg}$. After these therapeutic interventions, hypotension was reversed, and the patient showed gradual clinical and laboratory improvement.

$E$. coli was isolated in all sets of blood cultures and in urine culture that were taken on admission. Antimicrobial susceptibility testing was performed by the Kirby Bauer disc diffusion method [5]. The isolate was found to be resistant only to ampicillin (endogenous). Consequently, the treatment was deescalated and the patient was treated with intravenous ceftriaxone ( 2 g every $24 \mathrm{~h}$ ) and ciprofloxacin (400 mg every $12 \mathrm{~h}$ ). The patient had a successful clinical response to treatment with resolution of symptoms and signs of infection and improvement of relevant laboratory data within 4 days, while repeated blood cultures turned out negative from the fourth day of antimicrobial therapy. A repeated echocardiography, performed 

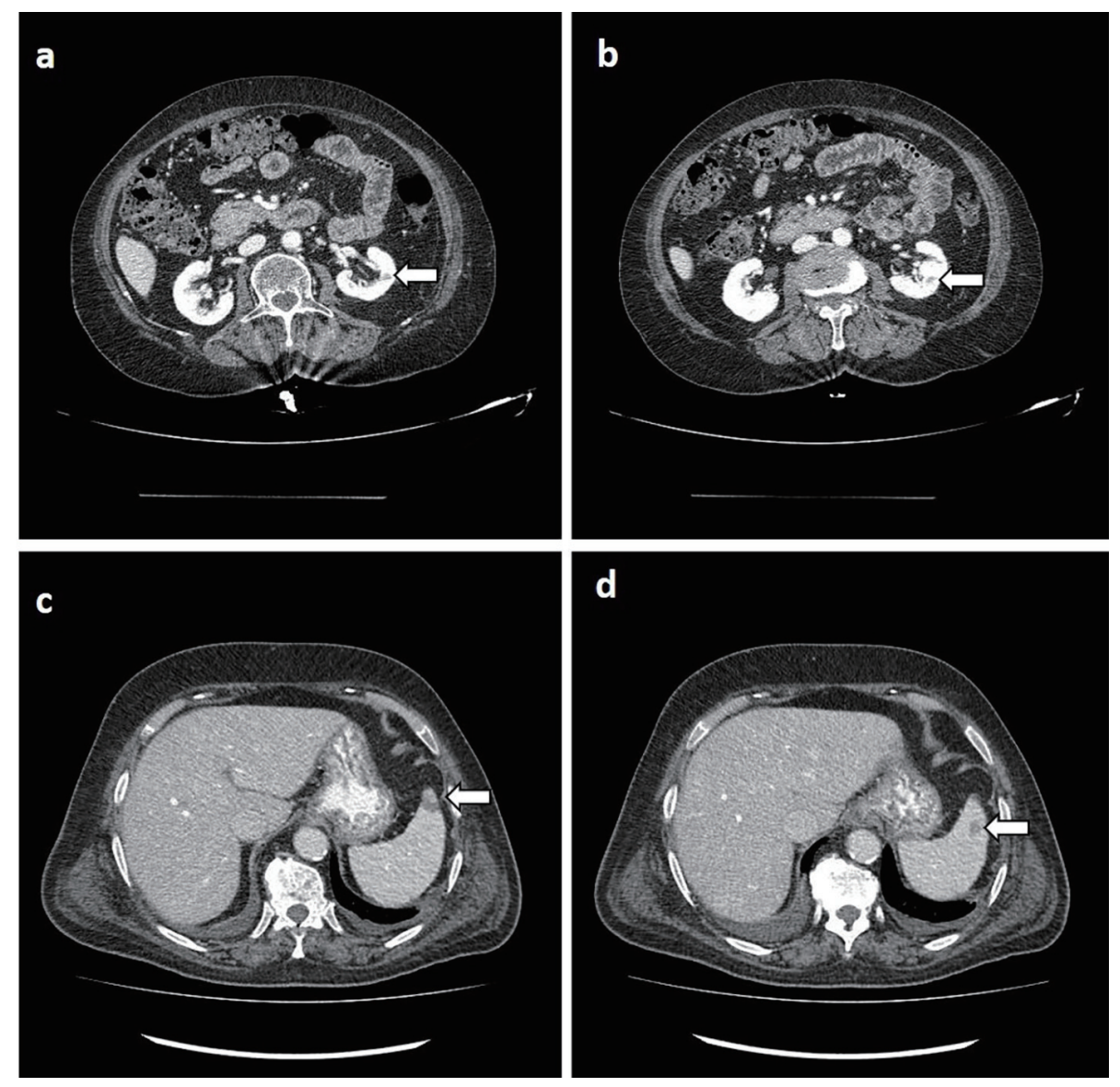

Figure 1. Contrast-enhanced abdominal computed tomography demonstrating infracts in left kidney (a, b, white arrows) and at the periphery of spleen (c, d, white arrows).

a week after patient's admission, showed improvement of left ventricular ejection fraction to $50 \%$, while mitral valve vegetation remained stable. After completion of 2 weeks of intravenous antibiotics, the patient was in very good clinical condition with normalization of all laboratory abnormalities (Table 1) and was referred for surgical management of endocarditis. $E$. coli was isolated from surgical specimens of replaced valve as well, despite previous clearance of blood cultures. Post-operatively, the patient continued antibiotic therapy with ceftriaxone and ciprofloxacin for additional 4 weeks and remained free of complications for a year at follow-up.

\section{Discussion}

$E$. coli has emerged in recent years as an increasingly important cause of morbidity and mortality in both immunocompetent and immunosuppressed people. In an 18-year prospective survey of 3.605 bacteremia episodes, E. coli accounted for 861 $(23.9 \%)$ cases and the most common site of infection was the urinary tract [6]. However, E. coli represents an uncommon etiologic factor of endocarditis accounting for a rate of about $0.5 \%$ of IE cases [4]. The low representativeness of $E$. coli as a cause of endocarditis, despite the high incidence of isola- tion of $E$. coli in bacteremias, is explained by its low ability to adhere to damaged or inflamed valves and to platelet-fibrin thrombus via their surface adhesins unlike Gram-positive bacteria (staphylococci, streptococci and enterococci) [7]. Previous studies have demonstrated that certain strains of $E$. coli are able to colonize tissue outside the gastrointestinal tract [3]. These strains, described as extra-intestinal pathogenic E. coli (ExPEC), predominantly belong to the phylogenetic group B2 and contain a variety of virulence factors that may enable the pathogens to invade and induce infections in the cardiac endothelium [8]. Administration of immunosuppressive therapy seems to constitute a risk factor for IE due to GNB, suggesting that a failure in the immune system could favor valve colonization [9]. The incidence of E. coli endocarditis increases with age and more than $70 \%$ of affected patients are females older than 70 years old [3]. Regarding advanced age, it is known that the cytotoxic responses of T-lymphocytes are decreased during senescence and therefore aging is a factor of relative immunosuppression (immunosenescence) [10]. Other risk factors for Gram-negative IE include implanted endovascular devices and invasive procedures in genitourinary and gastrointestinal systems $[4,11]$.

The presented case developed E. coli mitral valve endocarditis, presenting with septic shock and multiple organ 


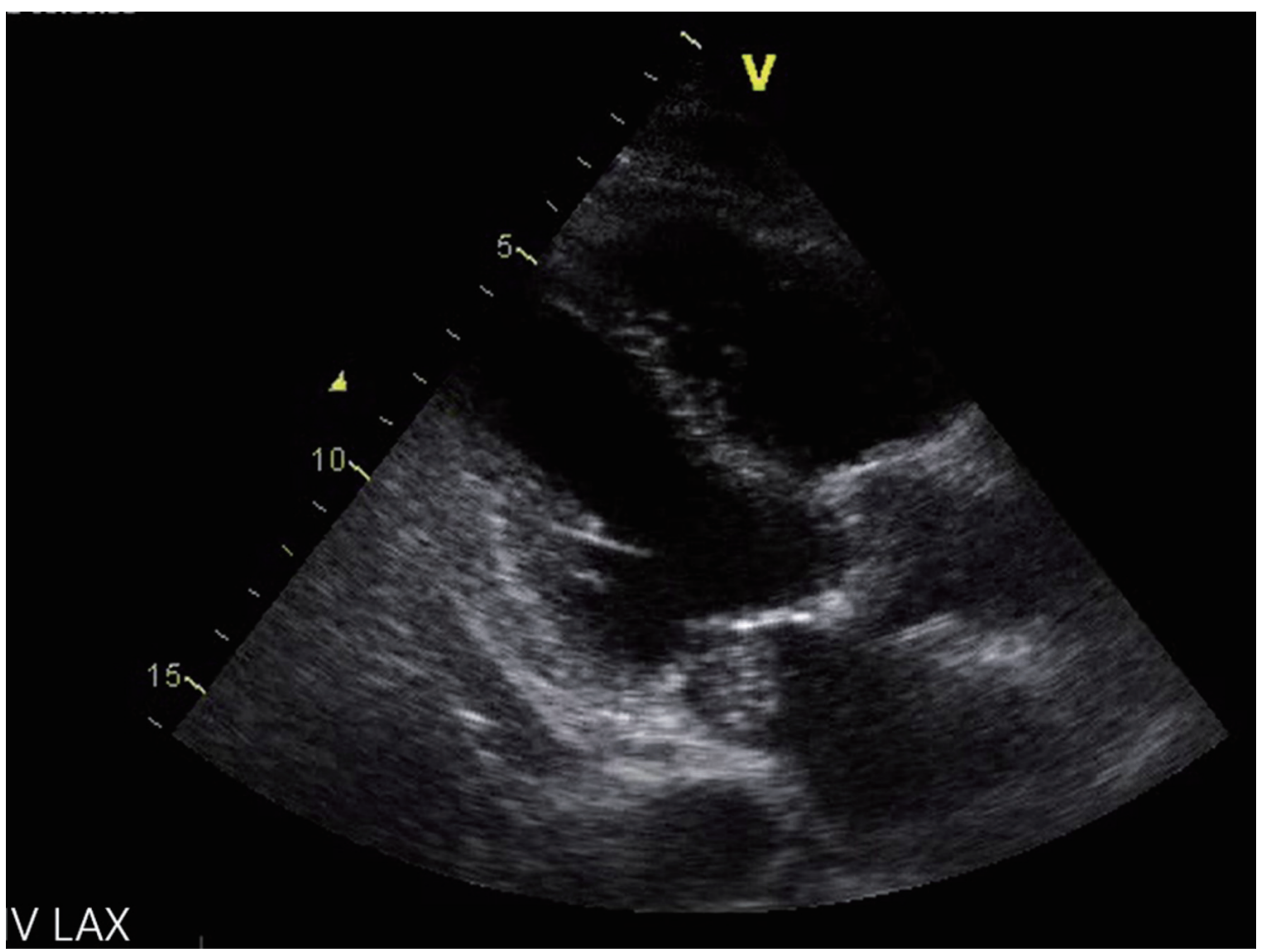

Figure 2. Echocardiography (parasternal long axis view): a large $(>15 \mathrm{~mm})$ mobile mass on the atrial side of the posterior mitral valve leaflet is seen (arrow).

dysfunction syndrome, in the absence of any underlying immunosuppression, except advanced age, or recently performed genitourinary or gastrointestinal invasive procedures. According to patient's initial symptoms (dysuria and fever with rigors) and positive urine culture, the urinary tract was the primary infectious focus. Diagnosis of endocarditis was based on Duke criteria; the patient fulfilled one major (endocardial involvement as evidenced by oscillating intra-cardiac mass) and three minor (fever, positive blood cultures with microorganisms not typical for IE, and arterial emboli) [1]. The presence of metastatic septic infracts involving spleen and left kidney made the diagnosis of endocarditis even more compelling. The mortality rates of E. coli IE are high; patients with IE due to GNB, prospectively observed in 26 Italian centers from 2004 to 2011, presented in-hospital mortality of $13.8 \%$, which rose to $30.6 \%$ at 1 year [2]. Gram-negative septic shock has a mortality rate of $12-38 \%$ [12-14]. The combination of the aforementioned diseases would reasonably result in a highly lethal clinical condition. The early institution of appropriate empiric antimicrobial therapy and hemodynamic resuscitation contributed majorly in the positive clinical outcome of the presented patient.

Beyond antimicrobial treatment, surgical management of such patients is important to improve survival. In our case, the patient met the following criteria for surgical treatment: IE mitral valve with large vegetations $(>10 \mathrm{~mm})$ in combination with embolic episodes. For that reason, she received antibiotic treatment for 2 weeks and then she was referred for valve replacement, completing her medical management with a postoperative 4-week course of antibiotics. Current guidelines for management of IE recommend that non-HACEK Gram-negative endocarditis should be managed by prolonged courses of combined antibiotic therapy and cardiac surgery [1]. The necessity of cardiac surgery was underlined in the presented patient by the fact that $E$. coli was isolated from surgical specimens of replaced valve as well, despite previous administration of 2 weeks of appropriate therapy and clearance of blood cultures.

In conclusion, despite $E$. coli endocarditis is a very rare condition, its incidence seems to be increasing in aged patients. A high level of suspicion is important to ensure timely diagnosis and therapy in such cases. In selected patients, as those with persistent Gram-negative bacteremia or severe sepsis/ septic shock, echocardiography is of paramount importance for the diagnosis of Gram-negative endocarditis and should be included in our diagnostic algorithm of patient's evaluation.

\section{Acknowledgments}

None to declare. 


\section{Financial Disclosure}

None to declare.

\section{Conflict of Interest}

None to declare.

\section{Informed Consent}

A written informed consent was obtained from the patient for publication of this case.

\section{Author Contributions}

SA presented the idea and designed the concept of the paper. $\mathrm{CD}$ and $\mathrm{ML}$ performed background search and co-wrote the paper. AM, CT and NK contributed to writing of specific parts of the manuscript. MM and SA critically revised the paper.

\section{References}

1. Baddour LM, Wilson WR, Bayer AS, Fowler VG, Jr., Tleyjeh IM, Rybak MJ, Barsic B, et al. Infective endocarditis in adults: diagnosis, antimicrobial therapy, and management of complications: A scientific statement for healthcare professionals from the American Heart Association. Circulation. 2015;132(15):1435-1486.

2. Falcone M, Tiseo G, Durante-Mangoni E, Ravasio V, Barbaro F, Ursi MP, Pasticci MB, et al. Risk factors and outcomes of endocarditis due to Non-HACEK Gram-negative bacilli: data from the prospective multicenter Italian endocarditis study cohort. Antimicrob Agents Chemother. 2018;62(4):e02208-17.

3. Micol R, Lortholary O, Jaureguy F, Bonacorsi S, Bingen E, Lefort A, Memain N, et al. Escherichia coli native valve endocarditis. Clin Microbiol Infect. 2006;12(5):401-403.
4. Morpeth S, Murdoch D, Cabell CH, Karchmer AW, Pappas P, Levine D, Nacinovich F, et al. Non-HACEK gram-negative bacillus endocarditis. Ann Intern Med. 2007;147(12):829-835.

5. The European Committee on Antimicrobial Susceptibility Testing (EUCAST). Breakpoint tables for interpretation of MICs and zone diameters. Version 7.1. EUCAST, 2017. http://www.eucast.org.

6. Gransden WR, Eykyn SJ, Phillips I, Rowe B. Bacteremia due to Escherichia coli: a study of 861 episodes. Rev Infect Dis. 1990;12(6):1008-1018.

7. Werdan K, Dietz S, Loffler B, Niemann S, Bushnaq H, Silber RE, Peters G, et al. Mechanisms of infective endocarditis: pathogen-host interaction and risk states. Nat Rev Cardiol. 2014;11(1):35-50.

8. Russo TA, Johnson JR. Proposal for a new inclusive designation for extraintestinal pathogenic isolates of Escherichia coli: ExPEC. J Infect Dis. 2000;181(5):1753-1754.

9. Gould K, Ramirez-Ronda CH, Holmes RK, Sanford JP. Adherence of bacteria to heart valves in vitro. J Clin Invest. 1975;56(6):1364-1370.

10. Weyand CM, Goronzy JJ. Aging of the immune system. Mechanisms and therapeutic targets. Ann Am Thorac Soc. 2016;13(Suppl 5):S422-S428.

11. Branger S, Casalta JP, Habib G, Collard F, Raoult D. Escherichia coli endocarditis: seven new cases in adults and review of the literature. Eur J Clin Microbiol Infect Dis. 2005;24(8):537-541.

12. Kang CI, Kim SH, Park WB, Lee KD, Kim HB, Kim $\mathrm{EC}, \mathrm{Oh} \mathrm{MD}$, et al. Bloodstream infections caused by antibiotic-resistant gram-negative bacilli: risk factors for mortality and impact of inappropriate initial antimicrobial therapy on outcome. Antimicrob Agents Chemother. 2005;49(2):760-766.

13. Kumar A, Roberts D, Wood KE, Light B, Parrillo JE, Sharma S, Suppes R, et al. Duration of hypotension before initiation of effective antimicrobial therapy is the critical determinant of survival in human septic shock. Crit Care Med. 2006;34(6):1589-1596.

14. Gikas A, Samonis G, Christidou A, Papadakis J, Kofteridis D, Tselentis Y, Tsaparas N. Gram-negative bacteremia in non-neutropenic patients: a 3-year review. Infection. 1998;26(3):155-159. 